\title{
Clinical trial: lubiprostone in patients with constipation- associated irritable bowel syndrome - results of two randomized, placebo-controlled studies
}

D. A. DROSSMAN*, W. D. CHEY†, J. F. JOHANSON\$, R. FASS§, C. SCOTT, R. PANAS \& R. UENO

*University of North Carolina Center for Functional Gl and Mobility Disorders, University of North Carolina at Chapel Hill, Chapel Hill, NC, USA; †University of Michigan Health System, Ann Arbor, MI, USA; †University of Illinois College of Medicine at Rockford, Rockford, IL, USA; §Southern Arizona Veterans' Administration Health Care System and University of Arizona Health Sciences Center, Tuscon, AZ, USA; $\uparrow$ Sucampo Pharmaceuticals, Inc., Bethesda, MD, USA

Correspondence to:

Dr D. A. Drossman, University of North Carolina Center for Functional GI and Mobility Disorders, University of North Carolina at Chapel Hill, 4150 Bioinformatics Building, Chapel Hill, NC 27599, USA.

Email: drossman@med.unc.edu

Publication data

Submitted 6 October 2008

First decision 11 October 2008

Resubmitted 29 October 2008

Accepted 30 October 2008

Epub Accepted Article 4 November 2008

\author{
SUMMARY \\ Background \\ Effective treatments for irritable bowel syndrome with constipation \\ (IBS-C) are lacking.
}

Aim

To assess the efficacy and safety of lubiprostone in IBS-C.

\section{Methods}

A combined analysis was performed among 1171 patients with a Rome II diagnosis of IBS-C in two phase- 3 randomized trials of lubiprostone $8 \mathrm{mcg}$ vs. placebo twice daily for 12 weeks. Using a balanced sevenpoint Likert scale ranging from significantly relieved $(+3)$, to significantly worse $(-3)$, patients responded on their electronic diary to the question: 'How would you rate your relief of IBS symptoms over the past week compared to how you felt before you entered the study?'. The primary efficacy endpoint was the percentage of overall responders.

\section{Results}

Using an intent-to-treat analysis with last observation carried forward, a significantly higher percentage of lubiprostone-treated patients were considered overall responders compared with those treated with placebo $(17.9 \%$ vs. $10.1 \%, P=0.001)$. Patients treated with lubiprostone reported a similar incidence of adverse events to those treated with placebo.

\section{Conclusions}

The percentage of overall responders based on patient-rated assessments of IBS-C symptoms was significantly improved in patients treated with lubiprostone $8 \mathrm{mcg}$ twice daily compared to those treated with placebo. Lubiprostone was well tolerated with a favourable safety profile.

Aliment Pharmacol Ther 29, 329-341 


\section{INTRODUCTION}

The irritable bowel syndrome (IBS) is defined by the presence of abdominal discomfort or pain in association with altered bowel habits (i.e. constipation, diarrhoea or a mixture of both). ${ }^{1}$ Of the approximately 30 million individuals in North America who meet the diagnostic criteria for IBS, approximately one-third experience constipation during episodes of disease activity. ${ }^{2}$ The remaining patients experience diarrhoea or mixed episodes between constipation and diarrhoea. $^{2,3}$ Several underlying mechanisms have been implicated in the pathophysiology of IBS, although much remains poorly understood. ${ }^{4,5}$ As a consequence, most currently available therapies are aimed at alleviating specific symptoms.

Lubiprostone is an orally active prostone that stimulates chloride secretion through activation of type-2 chloride channels (ClC-2) in the gastrointestinal tract. Through this action, lubiprostone enhances gastrointestinal fluid secretion and transit and improves the symptoms of constipation. ${ }^{6,7}$ Recent animal studies also suggest a role for this drug in stabilizing mucosal membranes, which may help reduce mucosal inflammation and membrane sensitization. ${ }^{8,9}$ Lubiprostone was approved in the US for the treatment of adults with chronic idiopathic constipation (CC) in 2006 and for the treatment of IBS with constipation (IBS-C) in adult females in 2008. ${ }^{10}$ In a post hoc analysis of patients from a pivotal phase- 3 trial of patients with CC who indicated IBS as a confounding condition, treatment with lubiprostone was associated with significant improvement in bowel movement frequency as well as symptoms of abdominal discomfort/pain and bloating as compared to placebo. ${ }^{11} \mathrm{~A}$ subsequent phase-2b dose ranging study also identified benefits of lubiprostone for IBS-C. ${ }^{7}$

Based on the encouraging results of the post hoc analysis from the pivotal CC trials and phase-2b IBS-C trial, two phase- 3 trials were designed to evaluate the efficacy and safety of lubiprostone $8 \mathrm{mcg}$ administered twice daily in patients with IBS-C.

\section{AIMS}

The objectives of this analysis were to demonstrate the efficacy and safety of 12-week administration of oral lubiprostone when compared to placebo for the treatment of IBS-C. Lubiprostone was evaluated at a daily dose of $16 \mathrm{mcg}$ ( $8 \mathrm{mcg}$ twice daily) vs. placebo (twice daily).

\section{METHODS}

\section{Patient population}

Two phase-3 double-blind, randomized, placebocontrolled trials (protocol numbers SIB-0431 and SIB0432) were conducted at multiple centres across the US. The protocols and all study procedures were approved by the Institutional Review Board at each centre and the studies were conducted in accordance with the World Medical Association Declaration of Helsinki, the International Conference on Harmonisation Tripartite Guideline for Good Clinical Practice and applicable federal and local regulations. Prior to performance of any study-related procedure, all patients enrolled in the studies received comprehensible information about the trial in which they would be participating and each signed an informed consent document.

Both clinical trials were registered at http://clinical trials.gov and received registration IDs NCT00380250, NCT00399542. Results are posted at http://clinical trials.gov under the same registration IDs. These trials have not been published in full elsewhere.

The inclusion criteria for the two studies were the same, with eligible patients being at least 18 years of age and meeting the Rome II Modular Questionnaire Criteria for Constipation-Predominant IBS. ${ }^{12}$ Patients 50 years or older were required to have had a colonoscopy performed within the previous 5 years and after the onset of IBS symptoms to rule out other possible diseases. Patients under the age of 50 years were required to have a sigmoidoscopy performed. Patients excluded from study participation were those who were unable or unwilling to use an acceptable method of birth control; females who were pregnant or nursing and those with a potential for noncompliance with the study protocol. Additional exclusion criteria included those with: previous gastrointestinal or abdominal surgery (except for common causes unrelated to IBS); organic disorder of the large or small bowel (e.g. ulcerative colitis, Crohn's disease); mechanical obstruction; unexplained significant weight loss or rectal bleeding; diagnosis of any medical condition associated with constipation (other than IBS); or conditions that might interfere with the conduct of the study, including other significant medical conditions, 
renal impairment, cancer, abnormal laboratory tests, recent abuse of alcohol or drugs, use of any medication indicated for treatment of IBS within the 4 weeks preceding randomization or use of investigational medications during the 4 weeks prior to screening.

\section{Study protocols}

The study designs are presented in Figure 1. For the purposes of this manuscript, the 'optional extension study' referred to in both schema will not be presented, nor will 'Treatment Period Phase II' of study 0431.

Patients meeting the inclusion criteria entered a 4week baseline/screening period that preceded each of the two studies. Those who did not have a flexible sigmoidoscopy or colonoscopy within the previous 5 years underwent the relevant endoscopic procedure during this period. During the 4-week screening period, patients were required to discontinue all laxative medications [over-the-counter (OTC) and prescription] and other specified disallowed medications for the duration of the study and were instructed to maintain a stable diet with no significant changes in their consumption of liquids or fibre or in their level of physical activity. During the screening period, patients accessed an electronic diary system on a daily basis to report the occurrence and time of all spontaneous bowel movements (SBMs), defined as those that occurred without the use of rescue medications. Patients used symptom scales previously utilized ${ }^{11}$ that included BM consistency (rated as $0=$ very loose, 1 = loose, $2=$ normal, $3=$ hard and $4=$ very hard), constipation severity, straining, abdominal bloating and discomfort (rated as $0=$ absent, $1=$ mild, $2=$ moderate, $3=$ severe and $4=$ very severe). If the patient did not experience a BM for 3 or more consecutive days and needed relief, the study investigator could authorize the patient to administer a rescue medication. Initial rescue treatment consisted of a $10 \mathrm{mg}$ Dulcolax (bisacodyl; Boehringer Ingelheim $\mathrm{GmbH}$, Ingelheim Rhein, Germany). If this was not successful a Fleet enema (dibasic sodium phosphate and monobasic sodium phosphate; C.B. Fleet, Lynchburg, VA, USA) could then be used. If both of these treatment options were unsuccessful, an alternative medication could be prescribed after consultation with the investigator.

At the end of the screening period, patients were assessed by medical history, physical examination, and laboratory tests. They completed an IBS quality of life questionnaire (IBS-QOL) as well. ${ }^{13}$ Daily diary data were reviewed and those who met the inclusion criteria, with $>70 \%$ completion of their electronic daily diary with these data indicating abdominal discomfort/pain with a monthly average assessment of mild or greater severity and any two of the following symptoms: <3 SBMs per week at least 25\% of the time, at least 25\% of SBMs recorded a straining assessment of moderate or greater severity, at least $25 \%$ of SBMs recorded a stool consistency assessment of hard or very hard stool, were eligible to enter the double-blind phases of their respective trial.

Patients enrolled in each trial were randomized to receive either lubiprostone $8 \mathrm{mcg}$ or placebo capsules using a computer-generated randomization scheme. Each drug was administered twice daily with breakfast and dinner with at least 8 ounces of water for a period of 12 weeks. A reduction to once-daily dosing was allowed, at the discretion of the investigator, if the patient experienced nausea or diarrhoea for more than 2 days or if patients experienced other adverse events. During treatment, patients were prohibited from taking prescription or OTC medications for constipation. Use of a daily fibre supplement was permitted, but patients were recommended to keep stable fibre therapy throughout the study period. Nonconstipation-related prescriptions and other OTC medications were allowed and their usage was documented as was as any medication change. As in the screening period, the use of rescue medications was allowed in those who failed to have a BM for 3 or more consecutive days and thus needed relief.

During the 12-week study phase, clinic visits were conducted at weeks 4,8 , and 12 and phone interviews were performed at weeks 1 and 2 following the final study visit. Patients continued to access the electronic diary system to report their SBMs, BM consistency and symptoms of constipation, medication usage and constipation rescue medications. On a weekly basis, patients also answered the electronic diary question: 'How would you rate your relief of IBS symptoms over the past week compared with how you felt before you entered the study?' using a seven-point balanced scale. At each clinic visit, the daily diary information was reviewed and discussed with the patient, adverse events were recorded, remaining capsules were collected to assess compliance and assessments of vital signs and laboratory parameters were performed. Patients also completed the IBS-QOL at week 4 and 12 visits. 
(a)

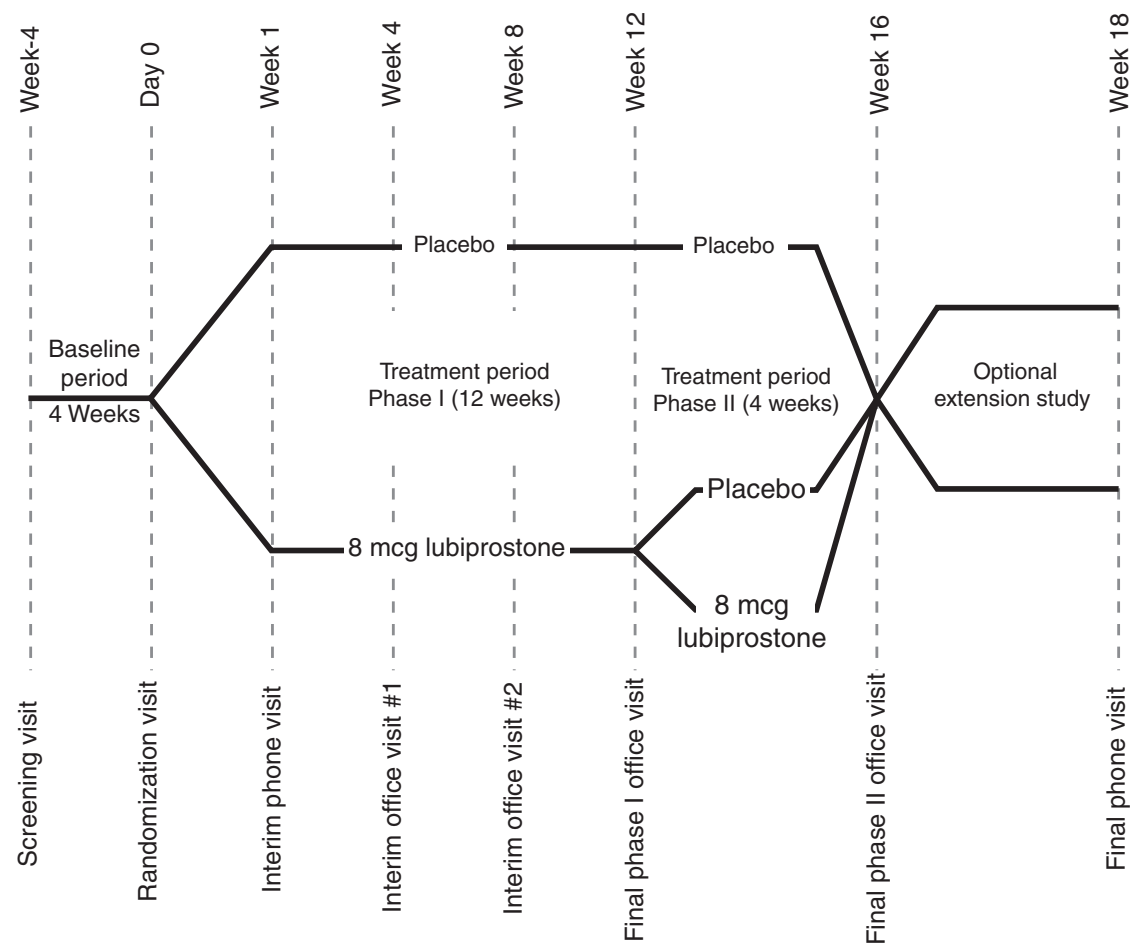

(b)

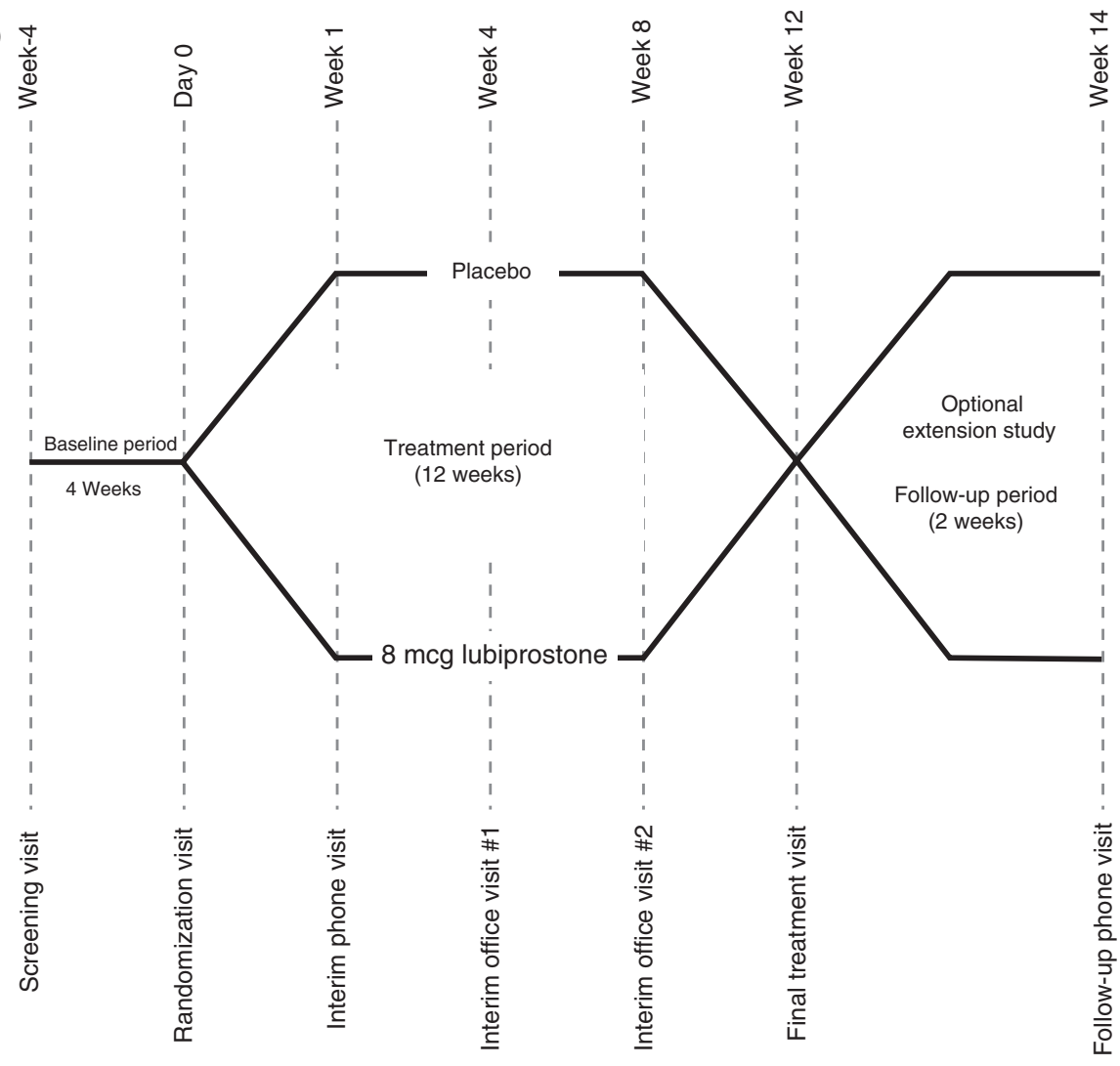

Figure 1. (a) Schematic of Study 0431. (b) Schematic of Study 0432. Note: For each study, the 'Optional Extension Study' methodology and results are not presented in this article. For study 0431, Treatment period Phase II methodology and results are not presented in this article. 


\section{Efficacy endpoints}

The primary efficacy endpoint of the studies and the combined analysis was the overall responder status in the lubiprostone and placebo groups. This endpoint was calculated from the weekly assessments of symptom relief gathered as part of the patient's electronic diary responses to the question 'How would you rate your relief of IBS symptoms over the past week compared to how you felt before you entered the study?' There were seven possible response options on a balanced scale that included 'Significantly worse', 'Moderately worse', 'A little bit worse', 'Unchanged', 'A little bit relieved', 'Moderately relieved' or 'Significantly relieved' (Figure 2). A weekly responder was defined as reporting either moderately or significantly relieved for that week. Monthly responders were defined as those who rated their IBS symptoms as being at least moderately relieved for all 4 weeks of the month or significantly relieved for at least 2 weeks of the month, with no ratings of moderately or severely worse. In addition, the patient could not discontinue treatment during the 4-week period due to a lack of efficacy, and the percent of days of rescue medication use did not increase compared to baseline. A patient was considered an overall responder (primary efficacy endpoint) if they were monthly responders for at least 2 of the 3 months of the study.

Secondary efficacy endpoints included the patient's monthly responder status, the weekly responder rate and the symptom rating changes from baseline for their abdominal discomfort/pain, bloating, BM and SBM frequency, stool consistency, degree of straining, constipation severity and symptom relief as rated on five-point Likert scales ranging from 0 (absent) to 4 (very severe). The assessment of stool consistency was scored on a scale of 0 (very loose) to 4 (very hard). Each patient's subjective evaluation of the efficacy of treatment was recorded weekly using a scale of 0 (not at all effective) to 4 (extremely effective). Rescue medication use was also recorded. The effects of treatment on health-related QOL were evaluated using a validated questionnaire for assessing IBS-QOL. ${ }^{13}$

\section{Safety and tolerability}

Safety endpoints included recording of all adverse events, clinical laboratory analyses at multiple timepoints, vital signs and physical examinations.

\section{Statistical analyses}

Each study was statistically powered in identical fashion. Within each study, 570 subjects were required in a 2:1 randomization (lubiprostone:placebo). This sample size within each study provided at least $88 \%$ statistical power to observe a relative $70.6 \%$ increase in overall responders for lubiprostone compared to placebo. All primary and secondary efficacy endpoint analyses reported were performed on an intent-to-treat (ITT) basis, which included all patients who received

\author{
Responder Question: \\ "How would you rate your relief of IBS symptoms (abdominal discomfort/pain, bowel \\ habits, and other IBS symptoms) over the past week compared to how you felt \\ before you entered the study?" \\ 7-Point balanced scale: \\ 7-Significantly relieved \\ 6-Moderately relieved \\ 5-A little bit relieved \\ 4-Unchanged \\ 3-A little bit worse \\ 2-Moderately worse \\ 1-Significantly worse \\ Monthly Responder: A response of "moderately relieved" or better in 4 out of 4 weeks \\ $\sim$ or \\ A response of "significantly releved" in 2 out of 4 weeks
}

Overall Responder: A monthly responder for at least 2 out of the 3 months of the trial

Figure 2. Rigorous responder definition. 
study drug and had data available for at least one efficacy endpoint. To accommodate potential variability in baseline values, we performed analyses using changes from baseline rather than raw values. Baseline values were defined as the average of all daily values during the 4-week screening/baseline period. Analyses of adverse events and other safety parameters included all patients who received at least one dose of study drug.

The study designs of 0431 and 0432 were similar in design and conducted in parallel; therefore, we present the studies' results as a combined analysis. The combined analysis also provides greater power for signal identification in safety and efficacy endpoints. The analyses of the combined data were stratified by study. A Cochran-Mantel-Haenszel test stratified by study was used to analyse overall and monthly responder rates. To test significance at a given month (months 1-3 only) while protecting the experiment-wise type I error rate of $\alpha=0.05$, a combination of closed and sequential testing procedures were performed. The procedure involved three steps. The method of analysis of each step is described as follows: For step 1, the number of responder months during the first 2 months was summed for each subject so that each subject received a score of 0,1 or 2 . If the CMS test resulted in a $P$ value $<0.05$, then the procedure advanced into step 2 . Otherwise, the procedure stopped. In step 2, months 1 and 2 were tested individually and simultaneously. Statistical significance was declared for any test that results in a $P$-value $<0.05$. If statistical significance was declared for both months 1 and 2, then the testing procedure advanced into step 3. Otherwise, the procedure stopped. In step 3, month 3 was tested individually. This stepwise procedure was applied individually to the studies and combined and both analyses are presented here.

The treatment effect from an analysis of covariance (Ancova) was used to test for differences between the treatment groups for all secondary symptom endpoints. The ANCOVA model controlled for study and the baseline value was used as a covariate. The Ancova model was used to examine the relationship between change in symptoms and response.

The IBS-QOL responses were scored according to the user's manual and scaled scores were used for all analyses. ${ }^{13}$ ANCOVA were used to analyse IBS-QOL responses.

All efficacy analyses with the exception of IBS-QOL used the last observation carried forward (LOCF) procedure to impute data for missing observations.

\section{RESULTS}

The subject disposition of both studies is presented in Figure 3. In total, 1171 patients in both studies were randomized to receive either lubiprostone or placebo after completing the 4-week screening/baseline period and satisfying the inclusion criteria. In both studies, four patients withdrew before receiving study medication and therefore were not included in either safety or efficacy analyses. A total of 13 patients withdrew after receiving study drug but before undergoing any postdosing evaluations. These patients were included in the safety, but not in the efficacy analysis. Thus, 1154 patients were included in the efficacy (ITT) analysis and 1167 were included in the safety analysis.

In both studies, patients were mostly female (91.6\%), Caucasian $(77.4 \%)$ and had a mean age of 46.6 years. At baseline, mean total abdominal scores for abdominal discomfort/pain (2.08; moderate), abdominal bloating (2.26; moderate), constipation severity $(2.23$; moderate), weekly SBM frequency (3.88), stool consistency (2.76; normal-hard) and straining (2.39; moderate) were similar between the lubiprostone and placebo groups. Only the category of age showed a statistically significant $(P<0.05)$ difference between lubiprostone and placebo groups, which was not deemed clinically meaningful. Baseline demographics and disease-specific characteristics are presented in Table 1 . There were no significant differences in baseline symptom scores between the two combined studies, thus permitting the combined analysis of data.

\section{Primary endpoint}

In a combined (ITT with LOCF) analysis, the total number of overall responders in the lubiprostone group $(17.9 \%)$ was significantly higher than that in the placebo group $(10.1 \% ; P=0.001)$. These results are depicted in Figure 4).

Individually, both studies showed a statistically significantly higher number of overall responders and significance at the first month as well as at other months with respect to responders who received lubiprostone treatment (Table 2).

The symptoms of abdominal discomfort/pain, bloating, constipation severity, stool consistency and straining correlated with responder status (Figure 5), indicating the validity of the seven-point balanced scale. On average, responders had a greater than one 


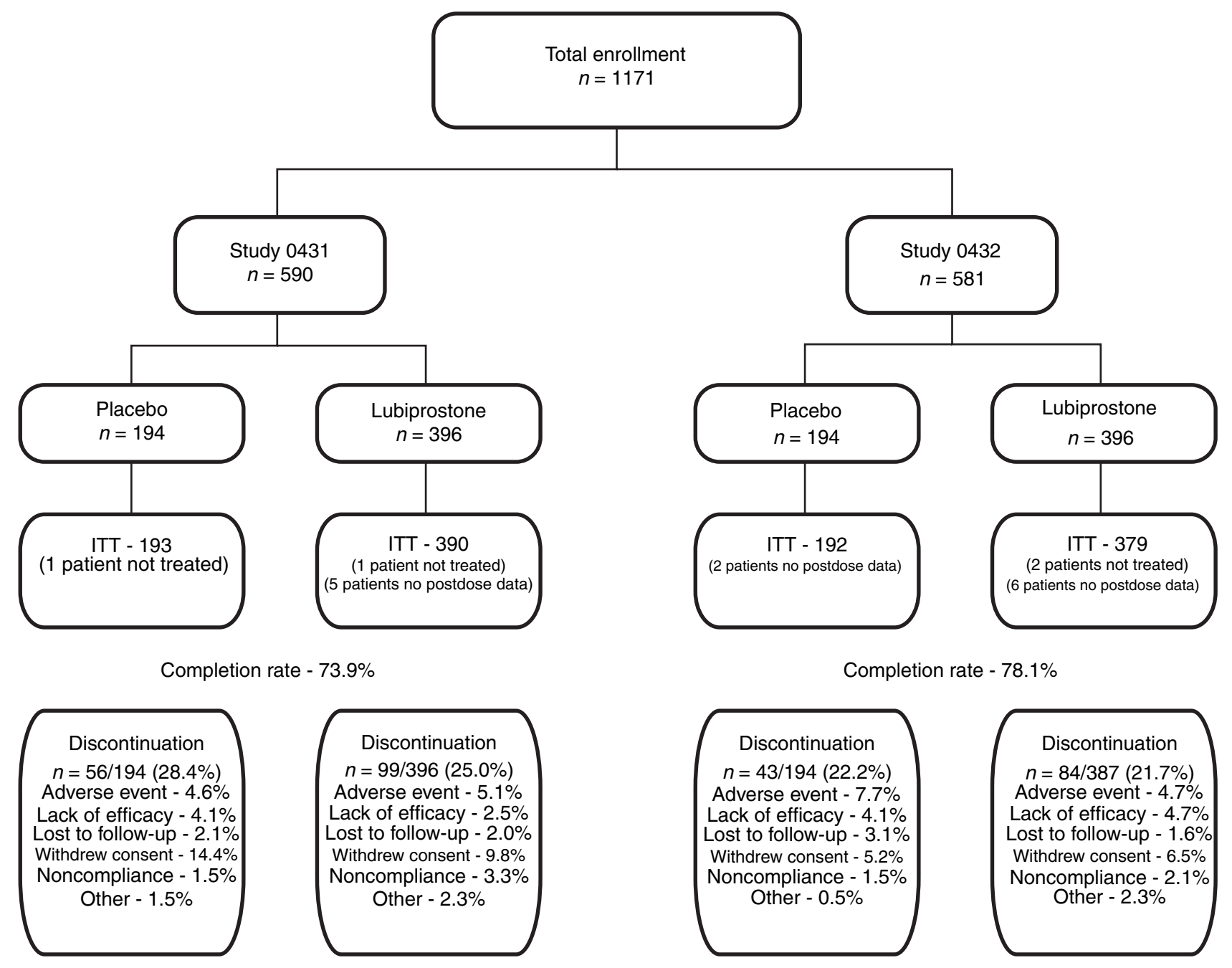

ITT - Intent-to-Treat Population

Figure 3. Patient disposition.

point reduction in their assessment of the IBS-C symptoms, whereas nonresponders showed only a one-third point reduction in their ratings. This also correlates with a clinically meaningful change (one point on a five-point scale, or a shift towards normal stools) ${ }^{14}$ in the responder group.

\section{Secondary endpoints}

The combined percentage of monthly responders using LOCF (Figure 6) was also significantly higher among those treated with lubiprostone compared with those treated with placebo at month 2 (18.3\% vs. $11.4 \%, P=0.003)$ and at month $3(22.0 \%$ vs. $14.5 \%, P=0.003)$. There was a trend towards a significantly higher percentage of monthly responders with lubiprostone compared with placebo at month $1(10.8 \%$ vs. 7.5\%, $P=0.078)$. For weekly responder rates, significant improvements were seen in the combined analysis with lubiprostone vs. placebo at weeks $2,4,5,6,10$ and $12(P \leq 0.030$; Figure 7).

Mean improvement from baseline in abdominal discomfort/pain was significantly greater in lubiprostonetreated patients compared with placebo-treated patients at month $2(-0.43$ vs. $-0.35, P=0.039)$ and month 3 
Table 1. Combined demographic and baseline characteristics of patients receiving at least one dose of study medication and having at least one postbaseline efficacy measurement

\begin{tabular}{|c|c|c|c|c|}
\hline \multirow[b]{2}{*}{ Parameter } & \multicolumn{2}{|l|}{ Treatment group } & \multirow[b]{2}{*}{ Total $(n=1154)$} & \multirow[b]{2}{*}{$P$-value } \\
\hline & Placebo $(n=385)$ & Lubiprostone ( $n=769$ ) & & \\
\hline \multicolumn{5}{|l|}{ Age (years) } \\
\hline$n$ & 385 & 769 & 1154 & $0.049^{*}$ \\
\hline Mean (min, max) & $47.7(18.0,85.0)$ & $46.1(19.0,83.0)$ & $46.6(18.0,85.0)$ & \\
\hline s.d. & 12.94 & 12.84 & 12.89 & \\
\hline \multicolumn{5}{|l|}{ Gender } \\
\hline Female & $359(93.2 \%)$ & $698(90.8 \%)$ & $1057(91.6 \%)$ & 0.152 \\
\hline Male & $26(6.8 \%)$ & $71(9.2 \%)$ & 97 (8.4\%) & \\
\hline \multicolumn{5}{|l|}{ Race } \\
\hline American Indian/Alaska Native & $0(0.0 \%)$ & $1(0.1 \%)$ & $1(0.1 \%)$ & 0.053 \\
\hline Asian & $2(0.5 \%)$ & $3(0.4 \%)$ & $5(0.4 \%)$ & \\
\hline Black/African American & $50(13.0 \%)$ & $102(13.3 \%)$ & $152(13.2 \%)$ & \\
\hline Caucasian & $298(77.4 \%)$ & $595(77.4 \%)$ & $893(77.4 \%)$ & \\
\hline Hispanic/Latino & $30(7.8 \%)$ & $68(8.8 \%)$ & $98(8.5 \%)$ & \\
\hline Other & $5(1.3 \%)$ & $0(0.0 \%)$ & $5(0.4 \%)$ & \\
\hline \multicolumn{5}{|l|}{ Abdominal discomfort/pain } \\
\hline$n$ & 385 & 769 & 1154 & 0.814 \\
\hline Mean (min, max) & $2.08(0.46,4.00)$ & $2.07(0.36,4.00)$ & $2.08(0.36,4.00)$ & \\
\hline s.d. & 0.667 & 0.658 & 0.661 & \\
\hline \multicolumn{5}{|l|}{ Abdominal bloating } \\
\hline$n$ & 385 & 769 & 1154 & 0.961 \\
\hline Mean (min, max) & $2.26(0.57,4.00)$ & $2.26(0.62,4.00)$ & $2.26(0.57,4.00)$ & \\
\hline s.d. & 0.694 & 0.684 & 0.687 & \\
\hline \multicolumn{5}{|l|}{ Constipation severity } \\
\hline$n$ & 385 & 769 & 1154 & 0.485 \\
\hline Mean (min, max) & $2.25(0.32,4.00)$ & $2.22(0.00,3.96)$ & $2.23(0.00,4.00)$ & \\
\hline s.d. & 0.645 & 0.661 & 0.655 & \\
\hline \multicolumn{5}{|l|}{ Weekly SBM frequency } \\
\hline$n$ & 385 & 769 & 1154 & 0.752 \\
\hline Mean (min, max) & $3.84(0.00,28.81)$ & $2.22(0.00,36.5)$ & $3.88(0.00,36.5)$ & \\
\hline s.d. & 3.571 & 3.320 & 3.405 & \\
\hline \multicolumn{5}{|l|}{ SBM stool consistency } \\
\hline$n$ & 364 & 745 & 1109 & 0.834 \\
\hline Mean (min, max) & $2.75(0.57,4.00)$ & $2.76(0.00,4.00)$ & $2.76(0.00,4.00)$ & \\
\hline s.d. & 0.690 & 0.658 & 0.668 & \\
\hline \multicolumn{5}{|l|}{ SBM bowel straining } \\
\hline$n$ & 364 & 745 & 1109 & 0.774 \\
\hline Mean (min, max) & $2.40(0.00,4.00)$ & $2.39(0.00,4.00)$ & $2.39(0.00,4.00)$ & \\
\hline s.d. & 0.742 & 0.699 & 0.713 & \\
\hline
\end{tabular}

SBM, spontaneous bowel movement.

* Statistically significant $(P \leq 0.05)$.

(-0.45 vs. $-0.36, P=0.028)$. Mean improvement from baseline in the lubiprostone group was significantly greater than the mean observed with placebo for abdominal bloating at month $2(P=0.044)$; BM frequency at month $1(P=0.021)$; stool consistency at months 1,2 and $3(P \leq 0.022)$; and degree of straining at months 1 and $2(P \leq 0.013)$ (Table 3$)$.

\section{Quality of life}

In the overall analysis of IBS-QOL, there was a trend towards greater improvement with lubiprostone at week $12(P=0.066)$. Analysis of subdomains demonstrated that 'body image' and 'health worry' were significantly improved in the lubiprostone group at week 


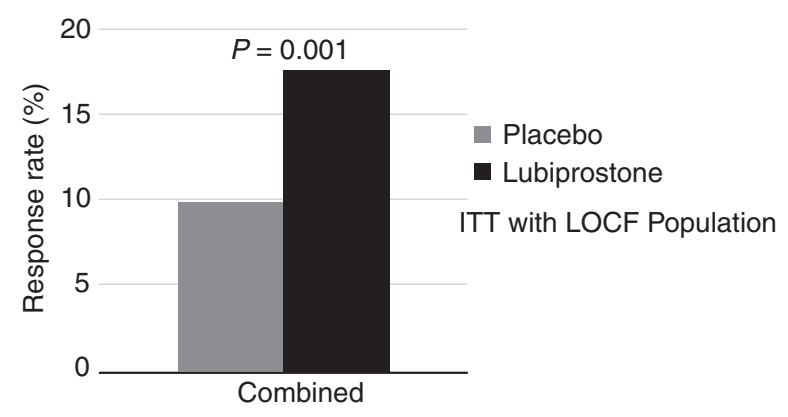

Monthly responder for $\geq 2 / 3$ months during treatment

Figure 4. Combined overall responder rate.

$12(P \leq 0.025)$. With a third domain, dysphoria, there was a trend for improvement in lubiprostone-treated patients, but this was not statistically significant $(P=0.086)$. Lubiprostone produced clinically meaningful changes ( $>14$ points) ${ }^{13}$ in the IBS-QOL domains of social reaction, food avoidance, health worry, body image and dysphoria.

\section{Safety and adverse events}

Overall, 588 (50\%) patients reported at least one adverse event (Table 4). There was a similar incidence of adverse events in both treatment groups: 50\% of lubiprostone and 51\% of placebo-treated patients reported at least one adverse event. Most adverse events were mild-to-moderate in intensity and resolved without intervention. The most frequently reported adverse events were related to the gastrointestinal tract (i.e. nausea, diarrhoea and abdominal distension) and occurred with similar incidence in both treatment groups $\quad$ (lubiprostone $=19 \%, \quad$ placebo $=$ 14\%). Patients who withdrew because of an adverse event are detailed in Figure 3. There were no significant differences between patients who withdrew from the lubiprostone groups and those in the placebo group.

A total of 11 serious adverse events (defined as those that were fatal, life-threatening, required hospitalization or intensive medical intervention) were reported during the trials, seven $(1 \%)$ in the lubiprostone group and four (1\%) in the placebo group. One serious adverse event was considered possibly related to lubiprostone, an episode of noncardiac chest pain in a 69-year-old female with a pre-existing cardiac medical history who was hospitalized one day after initiating treatment with lubiprostone. A cardiac workup ruled out cardiac cause, and the patient's chest pain resolved the next day. One patient randomized to the lubiprostone treatment arm died during the study. The patient, a 71-year-old male with a history of diabetes mellitus, hyperlipoidaemia and obesity, experienced a fatal cardiac arrest after 72 days of lubiprostone treatment. The investigator did not consider the patient's death as related to administration of lubiprostone given the patient's longstanding medical history.

No clinically significant differences between the two patient groups were detected in the analyses of laboratory values, vital signs or physical examination.

\section{DISCUSSION}

In the past, therapeutic modalities for IBS-C included bulking agents, osmotic laxatives and tegaserod, a partial 5-HT4 receptor agonist. Bulking agents may confer

Table 2. Overall and monthly responder rates by individual study (intent-to-treat, last observation carried forward population)

Treatment group (Study 0431)

Placebo $(n=193)(\%) \quad$ Lubiprostone $(n=390)(\%)$
Treatment group (Study 0432)

\begin{tabular}{|c|c|c|}
\hline -value* & $\begin{array}{l}\text { Placebo } \\
(n=192)(\%)\end{array}$ & $\begin{array}{l}\text { Lubiprostone } \\
(n=379)(\%)\end{array}$ \\
\hline
\end{tabular}

$\begin{array}{crc}\text { Timepoint } & & \\ \text { Overall } & 9.8 & 18.2 \\ \text { Month 1 } & 7.8 & 11.0 \\ \text { Month 2 } & 10.9 & 18.7 \\ \text { Month 3 } & 14.5 & 21.3\end{array}$

* $P$-values are from Cochran-Mantel-Haenszel tests stratified by centre. 


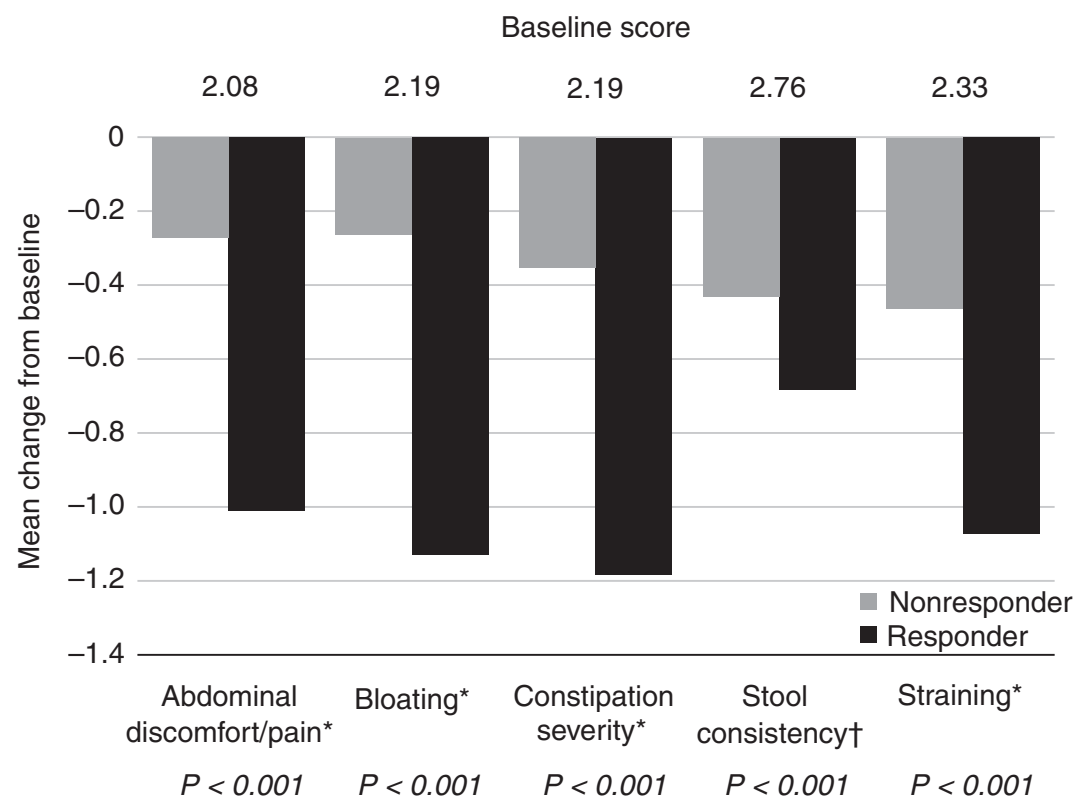

${ }^{*} 0$ (absent), 1 (mild), 2 (moderate), 3 (severe), 4 (very severe)

†0 (very loose [watery]), 1 (loose), 2 (normal), 3 (hard), 4 (very hard [little balls])

Figure 5. Lubiprostone symptom change: responder vs. nonresponder.

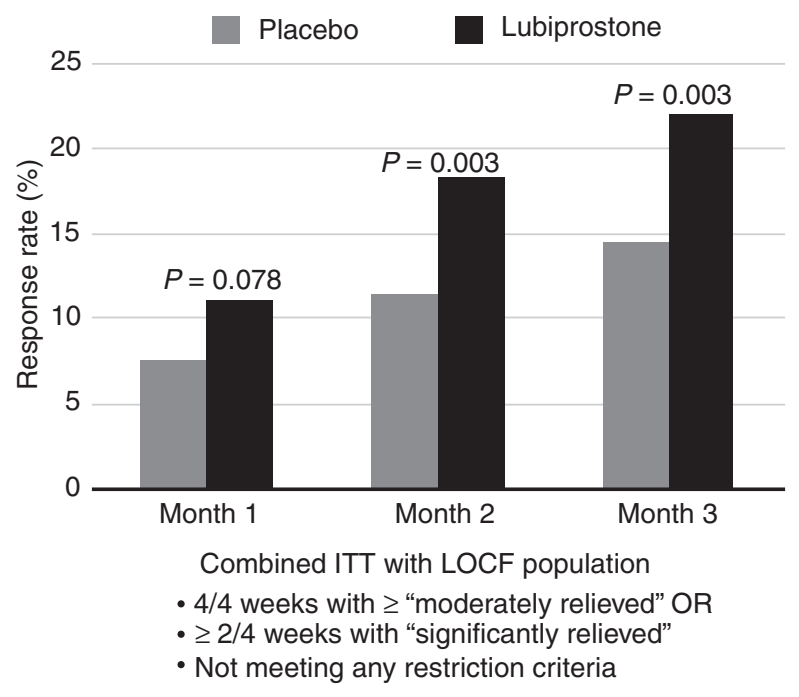

Figure 6. Combined monthly responder rate.

benefit to some patients, but are generally not more effective than placebo at relieving global IBS symptoms and may increase important IBS abdominal symptoms such as bloating. There exists very limited clinical trial evidence that supports the efficacy of osmotic laxatives in relieving abdominal discomfort or pain. ${ }^{15,16}$ Tegaserod has demonstrated clinical efficacy in ameliorating different IBS-related symptoms, but has recently been withdrawn in many countries

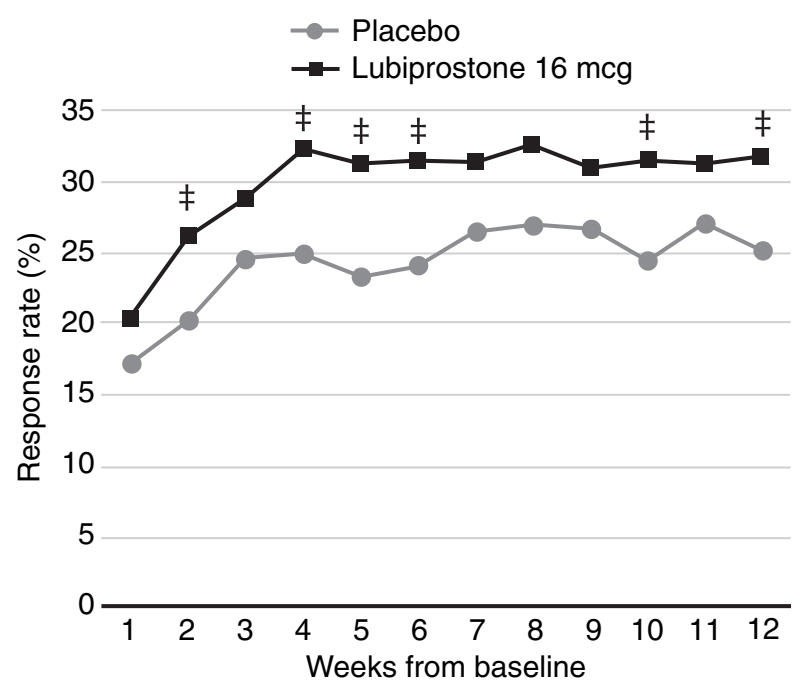

Figure 7. Combined weekly responder rate. $\ddagger P<0.05$.

because of concerns regarding cardiovascular safety issues. ${ }^{17}$ In the US, tegaserod is now only available through an emergency treatment investigational new drug application for patients with clinical circumstances, which are 'immediately life-threatening or serious enough to qualify for hospitalization'. ${ }^{18}$

The two trials described here contributed to FDA's approval in May 2008 of lubiprostone for the treatment of IBS-C in adult women. ${ }^{10}$ The results of 
Table 3. Combined secondary efficacy endpoint summary (intent-to-treat, last observation carried forward population)

\begin{tabular}{llll}
\hline Symptom measure & Month 1 & Month 2 & Month 3 \\
\hline Abdominal discomfort/pain & & $*$ & $*$ \\
Abdominal bloating & & $*$ & \\
Bowel movement Frequency & $*$ & & \\
Straining & $*$ & $*$ & $*$ \\
Constipation severity & $*$ & $*$ & $*$ \\
Stool consistency & $*$ & $*$ & $*$ \\
\end{tabular}

* Statistically significant $(P \leq 0.05)$.

Table 4. Combined summary of adverse events (safety population) (\%)

\begin{tabular}{lll}
\hline Safety-evaluable patients* & $\begin{array}{l}\text { Placebo } \\
(n=387)\end{array}$ & $\begin{array}{l}\text { Lubiprostone } \\
(n=779)\end{array}$ \\
\hline Serious adverse events & 1 & 1 \\
Treatment-related serious & 0 & $0.1 \dagger$ \\
adverse event & & \\
Treatment-related adverse events & 21 & 22 \\
$\quad$ Nausea & 4 & 8 \\
$\quad$ Diarrhoea & 4 & 6 \\
$\quad$ Abdominal distension & 2 & 2 \\
Study discontinuation & 7 & 5 \\
because of adverse event & & \\
\end{tabular}

* Includes all patients who received at least one dose of study medication.

$\uparrow$ One patient reported a noncardiac related chest pain that was deemed possibly because of lubiprostone.

the combined data from these two phase- 3 trials provide support for the efficacy of lubiprostone at a dosage of $8 \mathrm{mcg}$ twice daily in treating the global and individual symptoms experienced by patients with IBS-C. Lubiprostone significantly improved the chances of an IBS-C patient to become a responder (vs. a nonresponder) compared to placebo. Importantly, patients in the lubiprostone group had statistically significantly more (approximately twofold) treatment responders than those in the placebo group. These significant differences also occurred for the monthly responder rate at months 2 and 3 in both trials. Patients treated with lubiprostone experienced significant improvement in all secondary endpoints: abdominal discomfort/pain, bloating, straining and severity of constipation, as well as increased BM frequency and stool consistency. For most of these symptoms, the effect of lubiprostone persisted for the 3-month duration of the studies.

The relatively low response rates reported in the lubiprostone and placebo groups for the primary endpoint deserve discussion. These studies utilized a novel, highly rigorous responder definition, which was developed in conjunction with the US Food and Drug Administration. To be considered a monthly responder, patients had to report moderate relief of their IBS symptoms for 4 of 4 weeks or significant relief for more than 2 of 4 weeks. To be an overall responder, patients had to be a monthly responder for 2 of 3 months of the randomization period. It is possible that this highly rigorous endpoint may have underestimated the proportion of patients whom a practicing clinician would have considered successfully treated. For example, patients who reported moderate relief of their IBS symptoms for 3 of 4 weeks were not considered a monthly responder using this endpoint. Similarly, those with moderate relief of their IBS symptoms for 2 weeks and significant relief for 1 week were not considered a monthly responder. Furthermore, these studies' responder definition included not only patient self-assessment but also disallowed increased rescue medication use and study discontinuation because of lack of treatment efficacy. The rigour of the primary endpoint is highlighted by the placebo response of $\sim 10 \%$, a value lower than has been reported in previous IBS treatment trials. ${ }^{19}$ Despite the rigour of the primary endpoint, patients randomized to lubiprostone were nearly twice as likely to experience an overall improvement in IBS symptoms compared to those receiving placebo.

These current studies further demonstrate the benefit of lubiprostone in treating the constipation-associated symptoms of IBS. It is likely that these clinical benefits are mediated through the activation of ClC-2 channels ${ }^{20,21}$ leading to increased fluid secretion into the intestinal lumen. Furthermore, the activation by lubiprostone of $\mathrm{ClC}-2$ channels has been shown in animal studies to initiate restoration of tight junctions, leading to recovery of mucosal barrier function. $^{9,22}$ Recent studies suggest that increased intestinal permeability may be a contributing factor to the IBS clinical state. ${ }^{23}$ Thus, it is attractive to speculate that reduced intestinal permeability associated with an increased inflammatory response ${ }^{23-26}$ may enable the development of visceral hypersensitivity that is often present in patients with IBS. 
Possibly, lubiprostone may inhibit this effect in IBS which in turn may explain the improved global symptoms and pain scores. This finding may have important clinical implications for IBS-C as other anticonstipation agents like polyethylene glycol do not show pain benefit. ${ }^{27}$

Overall, lubiprostone at a dose of $8 \mathrm{mcg}$ twice daily was well-tolerated. There were no significant differences between the lubiprostone and placebo groups in reporting adverse events or serious adverse events. The most frequently-reported AEs were gastrointestinal-related and included nausea (8\% with lubiprostone and 4\% with placebo), diarrhoea (6\% with lubiprostone and 4\% with placebo) and abdominal distension (2\% in both groups). With respect to the nausea, most events were mild or moderate in nature, were much less frequent compared with higher dosages used for treating chronic constipation and resolved without intervention. The higher rate of diarrhoea in the lubiprostone group is consistent with the drug's mechanism of action. ${ }^{21}$ The reason for the higher incidence of nausea among IBS-C patients receiving lubiprostone is unknown, but consistent with results seen in previous chronic constipation studies. ${ }^{7,} 11$ The only serious adverse event that was considered possibly related to treatment, noncardiac chest pain in a patient with a pre-existing cardiac medical history, resolved within $24 \mathrm{~h}$. In previous clinical trials of constipated adults receiving $24 \mathrm{mcg}$ lubiprostone twice daily, no treatmentrelated cardiac deaths or serious adverse events have been reported. ${ }^{7,11}$

Based on results from a previous phase- 2 trial, ${ }^{7}$ lubiprostone significantly improved gastrointestinal symptoms of IBS-C across three dosing groups (8, 16 and $24 \mathrm{mcg}$ twice daily each) and the benefit in terms of pain was similar across all doses. The study determined that the $8 \mathrm{mcg}$ twice daily dose of lubiprostone demonstrated the optimal combination of efficacy and safety for IBS-C. The results of this combined analysis of two phase- 3 trials confirm the findings of the earlier phase-2 trial, with significant improvement in IBS-C symptoms reported for most parameters and a demonstrated an acceptable safety profile. For reasons of the unique primary endpoint response definition, the results are not comparable across IBS trials using a different primary endpoint definition.

In these 2 trials, lubiprostone treatment was associated with a trend towards improvement in overall
QOL. Furthermore, statistically significant improvements were seen in key subdomains (body image, health worry) with lubiprostone as compared to placebo. The overall trend was not seen in the previous phase-2 study, where no treatment differences were seen. The significant health worry improvement with lubiprostone was also seen in the previous trial.

\section{CONCLUSION}

The combined analysis of these two phase- 3 trials demonstrated that treatment with lubiprostone led to a significantly higher number of global responders and significantly improved individual symptoms in patients with IBS-C compared with placebo. The clinical benefits of lubiprostone were generally sustained over 12 weeks of the treatment period. Treatment with lubiprostone was associated with a similar overall incidence of adverse events as placebo; most of the events were gastrointestinal in nature, mild-tomoderate in intensity and short-lived. The frequency of serious adverse events and patient withdrawals from the study because of adverse events was similar with lubiprostone and placebo. From these data, we conclude that lubiprostone at a dose of $8 \mathrm{mcg}$ administered twice daily provides an efficacious and well tolerated treatment option for patients with IBS-C.

\section{ACKNOWLEDGEMENTS}

Declaration of personal interests: D. A. Drossman is a consultant and has received research funding from Takeda Pharmaceuticals North America, Inc. and is a consultant for Sucampo Pharmaceuticals, Inc. J. F. Johanson is on the speaker bureaux of and is a consultant for Takeda Pharmaceuticals North America, Inc. and Sucampo Pharmaceuticals, Inc. J. F. Johanson owns stock options in Sucampo Pharmaceuticals, Inc. W. D. Chey is on the speaker bureau of Takeda Pharmaceuticals North America, Inc. and has received research funding from Sucampo Pharmaceuticals, Inc. R. Fass receives research funding from Takeda Pharmaceuticals North America, Inc. and Sucampo Pharmaceuticals, Inc. R. Ueno is an employee of Sucampo Pharmaceuticals, Inc. and a shareholder in this company. R. Panas and A. Wahle are employees of Sucampo Pharmaceuticals, Inc. Declaration of funding interests: These studies were funded, designed, conducted and supervised in full by the sponsor, Sucampo 
Pharmaceuticals, Inc., Bethesda, MD. They were performed in compliance with Good Clinical Practice, including the archiving of essential documents. Sucampo Pharmaceuticals, Inc. analysed and interpreted the studies' data and oversaw the writing of the study reports. The Principal Investigator, D. A. Drossman, served as the guarantor of the submission. He and co-investigators were responsible for analysis and interpretation of the data and are the guarantors of the manuscript and its contents. All authors were involved in the development of the draft document and review of subsequent revisions. The writing of this manuscript, and subsequent writing and editorial support in the subsequent revisions, was supported in part by Sucampo Pharmaceuticals, Inc. and in part by Takeda Pharmaceuticals North America, Inc.

\section{REFERENCES}

1 Drossman DD, Corazziari E, Delvaux M, et al. Rome III: The Functional Gastrointestinal Disorders, 3rd edn. McLean, VA: Degnon Associates, Inc., 2006.

2 Drossman DA, Morris $\mathrm{CB}, \mathrm{Hu} \mathrm{Y}$, et al. A prospective assessment of bowel habits in irritable bowel syndrome in women: defining an alternator. Gastroenterology 2005; 128: 580-9.

3 Saito YA, Schoenfeld P, Locke GR III. The epidemiology of irritable bowel syndrome in North America: a systematic review. Am J Gastroenterol 2002; 97: 1910-5.

4 Camilleri M, Choi MG. Review article: irritable bowel syndrome. Aliment Pharmacol Ther 1997; 11: 3-15.

5 Drossman DA, Camilleri M, Mayer EA, Whitehead WE. AGA technical review on irritable bowel syndrome. Gastroenterology 2002; 123: 2108-31.

6 Lacy BE, Campbell LL. Lubiprostone: a chloride channel activator. J Clin Gastroenterol 2007; 41: 345-51.

7 Johanson JF, Ueno R. Lubiprostone, a locally acting chloride channel activator, in adult patients with chronic constipation: a double-blind, placebo-controlled, dose-ranging study to evaluate efficacy and safety. Aliment Pharmacol Ther 2007; 25: 1351-61.

8 Cuppoletti J, Malinowska DH, Tewari KP, et al. SPI-0211 activates T84 cell chloride transport and recombinant human $\mathrm{ClC}-2$ chloride currents. Am J Physiol Cell Physiol 2004; 287: C1178-83.

9 Moeser AJ, Nighot PK, Engelke KJ, Ueno R, Bilkslager AT. Recovery of mucosal barrier function in ischemic porcine ileum and colon is stimulated by a novel agonist of the $\mathrm{ClC}-2$ chloride channel, lubiprostone. Am J Physiol Gastrointest Liver Physiol 2007; 292: 647-56.
10 Amitiza [package insert]. Bethesda, MD: Sucampo Pharmaceuticals, Inc., 2008.

11 Johanson JF, Morton D, Geenen J, Ueno R. Multicenter, 4-week, double-blind, randomized, placebo-controlled trial of lubiprostone, a locally-acting type- 2 chloride channel activator, in patients with chronic constipation. Am J Gastroenterol 2008; 103: 170-7.

12 Drossman DD, Corazziari E, Delvaux M, et al. The Functional Gastrointestinal Disorders, 2nd edn. McLean, VA: Degnon Associates, Inc., 2000.

13 Drossman D, Morris CB, Yuming H, et al. Characterization of health related quality of life (HRQOL) for patients with functional bowel disorder (FBD) and its response to treatment. Am J Gastroenterol 2007; 102: 1-12.

14 Camilleri M, Mangel AW, Fehnel SE, Drossman DD, Mayer EA, Talley N. Primary endpoints for irritable bowel syndrome trials: a review of performance of endpoints. Clin Gastro Hepatol 2007; 5: 534-40.

15 Drossman DA, Camilleri M, Mayer EA, Whitehead WE. AGA technical review on irritable bowel syndrome. Gastroenterology 2002; 123: 2108-31.

16 American College of Gastroenterology Functional Gastrointestinal Disorders Task Force. Evidence-based position statement on the management of irritable bowel syndrome in North America. Am J Gastroenterol 2002; 97: S1-5.

17 Brandt LJ, Bjorkman D, Fennerty MB, et al. Systematic review on the management of irritable bowel syndrome in North America. Am J Gastroenterol 2002; 97: S7-26.

18 US Food and Drug Administration. Zelnorm (tegaserod maleate) Information. 2008. US FDA Web page: http:// www.fda.gov/cder/drug/infopage/zelnorm/ default.htm (accessed 30 September 2008).

19 Patel SM, Stason WB, Legedza A. The placebo effect in irritable bowel syndrome trials: a meta-analysis. Neurogastroenterol Motil 2005; 17: 332-40.

20 Mohammad-Panah R, Gyomorey K, Rommens J, et al. ClC-2 contributes to native chloride secretion by a human intestinal cell line, CACO-2. Biol Chem 2001; 276: 8306-13.

21 Cuppoletti J, Malinowska DH, Tewari KP, et al. SPI-0211 activates T84 cell chloride transport and recombinant human $\mathrm{ClC}-2$ chloride currents. Am J Physiol Cell Physiol 2004; 287: C1173-83.

22 Blikslager AT, Moeser AJ, Gookin JL, Jones SL, Odle J. Restoration of barrier function in injured intestinal mucosa. Physiol Rev 2007; 87: 545-64.

23 Camilleri M, Gorman H. Intestinal permeability and irritable bowel syndrome. Neurogastroenterol Motil 2007; 19: 545-52.

24 Dunlop SP, Hebden J, Campbell E, et al. Abnormal intestinal permeability in subgroups of diarrhea-predominant irritable bowel syndromes. Am J Gastroenterol 2006; 101: 1288-94.

25 Marshall JK, Thabane M, Garg AX, Clark W, Meddings J, Collins SM. Intestinal permeability in patients with irritable bowel syndrome after a waterborne outbreak of acute gastroenteritis in Walkerton, Ontario. Aliment Pharmacol Ther 2004; 20: 1317-22.

26 Spiller RC. Role of infection in irritable bowel syndrome. J Gastroenterol 2007; 42(Suppl. XVII): 41-7.

27 Khoshoo V, Armstead C, Landry L. Effect of a laxative with and without tegaserod in adolescents with constipation predominant irritable bowel syndrome. Ailment Pharmacol Ther 2006; 23: 191-6. 